\title{
Geração de planos de produção via otimização seqüencial subótima
}

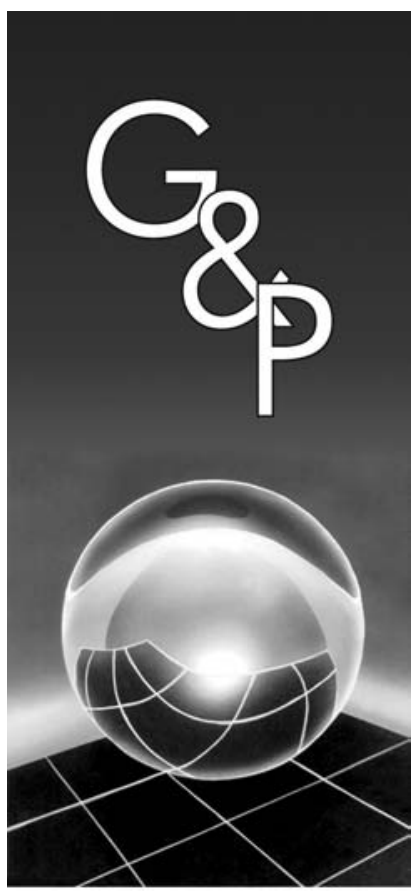

Oscar Salviano Silva Filho

Wagner Cezarino

\section{Resumo}

Neste artigo são discutidos aspectos relacionados à tomada de decisão dentro de um processo hierárquico de planejamento da produção. Neste contexto, foi desenvolvido um modelo agregado para um problema de planejamento estocástico, com restrições de chances nas variáveis de decisão, que serve como meta de produção a ser atingida nos níveis inferiores da hierarquia. Solução ótima global para este tipo de problema seqüencial é muito difícil, a menos em casos de pequena dimensão via algoritmo de programação dinâmica estocástica. Assim, como alternativa, investigou-se a utilização de uma heurística subótima denominada open-loop feedback controller (OLFC), que é muito simples de ser implementada computacionalmente. Como exemplo de aplicação, um problema mono-produto de planejamento agregado da produção foi formulado e a política subótima OLFC foi comparada à solução ótima usando o algoritmo de programação dinâmica estocástica. Além dos aspectos numéricos, algumas propriedades são analisadas, como a quase adaptabilidade do procedimento OLFC.

Palavras-chave: Controle subótimo. Modelos probabilísticos. Programação dinâmica. Estoques.

\section{Introdução}

Muitas decisões tomadas em um processo de planejamento são usadas para corrigir ou ajustar os recursos industriais de uma organização com respeito às metas pré-estabelecidas pela alta gerência, ou seja, os chamados planos agregados de produção, marketing, vendas, finanças, etc. Em particular, o plano agregado de produção assume um papel fundamental na construção das metas estratégicas a serem seguidas durante a realização das atividades de planejamento de médio e curto prazo. De fato, este plano apresenta-se como uma espécie de "guarda-chuva" que cobre, como um todo, os diferentes níveis hierárquicos que compõem o processo de planejamento da produção de uma organização (HAX; CANDEA, 1984).

O plano é, via de regra, feito sobre um horizonte de planejamento de 1 a 2 anos com discretização mensal. Devido às incertezas relacionadas às flutuações de demanda e às restrições físicas do processo de produção, o plano necessita ser periodicamente revisado para se ajustar às mudanças do ambiente produtivo. Deste modo, o plano torna-se uma meta realista a ser perseguida nas ações de planejamento desenvolvidas pelos níveis inferiores do processo hierárquico de decisões (SILVA FILHO, 1999).
No sentido de ilustrar o exposto acima, a Figura 1 mostra as principais interações existentes entre os níveis estratégico, tático e operacional do esquema clássico hierárquico de tomada de decisão, proposto por Antony (1965). Note que tais interações sugerem uma contínua e multidirecional troca de informações entre cada um dos níveis da hierarquia. Isto permite que as decisões tomadas, em um dado período de tempo, possam ser revisadas ou ajustadas, sempre que inconsistências na aplicação do plano de produção ao "mundo real" sejam detectadas.

Ainda com respeito à Figura 1, é interessante imaginar que existe um intenso fluxo de informação integrando continuamente os três níveis hierárquicos de tomada de decisão. Por exemplo, no nível estratégico, no qual se realiza o plano de produção, o volume de informação é considerado de forma agregada, ou seja, itens e produtos fabricados, com características similares, são considerados agrupados dentro de famílias. Com isto, pode-se reduzir significativamente a quantidade de dados a serem usados nas formulações matemáticas. Esta característica faz com que uma parte dos problemas tratados no nível estratégico seja do tipo bem-estruturados (GERSHWIN et al., 1986). Com isto, procedimentos e técnicas da 


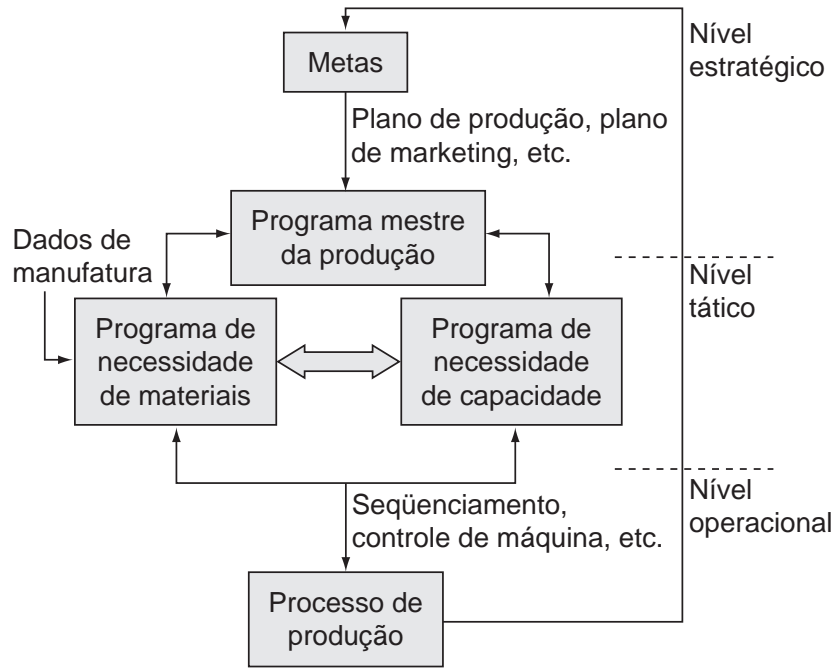

Figura 1. Fluxo de informações na cadeia hierárquica de três níveis de decisão.

programação matemática e da teoria de controle podem ser aplicados conjuntamente para solução de tais problemas (BENSOUSSAN et al., 1978; HAX; CANDEA, 1984; GERSHWIN et al., 1986).

O que se pode concluir do exposto acima é que a natureza dinâmica estocástica do ambiente organizacional, sujeita a limites operacionais de capacidade, influencia fortemente o desenvolvimento do plano agregado de produção. Isto significa que o plano deve ser gerado a partir de um modelo que leve em conta esta natureza e que também permita incorporar ajustes periódicos devido à captação de novas informações provenientes dos níveis inferiores, como ilustrado na Figura 1.

Dentro do contexto acima, um modelo de otimização seqüencial, não-linear, estocástico com restrições probabilísticas nas variáveis de decisão, pode ser considerado para representar o problema de geração de planos agregados de produção. É interessante comentar que esse modelo é representativo de uma ampla classe de problemas encontrados na prática. Na área gerencial, por exemplo, pode-se destacar além dos clássicos problemas de estoque-produção, problemas de planejamento envolvendo assuntos relacionados a finanças, marketing e vendas, dentre outros (BENSOUSSAN et al., 1978; NECK, 1984).

\subsection{Plano de produção via modelos seqüenciais estocásticos}

Como ilustrado na Figura 1, o plano de produção representa uma das primeiras atividades de planejamento em uma cadeia hierárquica de decisões. Na sua formulação mais simples, o problema de geração de um plano de produção consiste em determinar quais os níveis ótimos agregados de estoque e produção que devem ser disponibilizados para satisfazer a demanda dentro de um horizonte de longo prazo.

Algumas características importantes relacionadas a este tipo de problema são sucintamente apresentadas a seguir:

a) no longo prazo, a flutuação de demanda ao longo do tempo é aleatória e seu valor deve ser estimado com base em informações estatísticas. Uma imediata conseqüência disto é que a equação de balanço de estoque é um processo estocástico, sendo os níveis de estoque variáveis aleatórias;

b) a existência de fatores endógenos - como, por exemplo, atrasos de materiais, quebras de máquinas, etc - faz com que os níveis de estoques precisem ser constantemente atualizados (ou revisados);

c) se for considerado que variável de produção depende diretamente da variável de estoque, então esta variável terá de ser considerada como uma variável aleatória;

d) os níveis de estoques e produção estão restritos a tomarem valores de "espaços físicos" relacionados à capacidade de armazenagem e de produção, respectivamente; $\mathrm{e}$

e) as relações de custo, envolvendo as variáveis de estoque e produção, podem assumir características não lineares. Um caso muito comum na literatura é o emprego de modelos com custos quadráticos (HAX; CANDEA, 1984).

Com base nas características acima, é possível concluir que para geração de um plano de produção é preciso formular um modelo de otimização não linear seqüencial estocástico com restrições probabilísticas nas variáveis de decisão.

De modo geral, modelos analíticos baseados em programação estocástica podem ser utilizados para representar esta classe de problemas de planejamento agregado da produção (MULA et al., 2006). No entanto, tais modelos são muito complexos e não incorporam explicitamente o componente tempo, fornecendo, deste modo, apenas soluções estáticas, ou seja, congeladas no tempo. Exceção à regra é o clássico procedimento da programação dinâmica estocástica. De fato, este procedimento leva em conta o tempo no processo de tomada de decisão, porém torna-se inviável para aplicações práticas devido ao elevado requisito de armazenagem e tempo de processamento computacional (BERTESEKAS, 2000).

As dificuldades descritas acima têm levado a pesquisa por modelos seqüenciais mais simples (CHENG et al., 2004). Com tais modelos é possível reduzir a complexidade do problema original permitindo, assim, a geração de soluções computacionalmente mais eficientes (SAHINIDIS, 2004). Além disso, é possível combinar métodos da programação matemática com os da teoria de controle ótimo, produzindo procedimentos subótimos que 
podem ser aplicados ao modelo simplificado (BERTESEKAS, 2000; NECK, 1984; PEKELMAN; RAUSSER, 1978; BRYSON; HO, 1975).

\subsection{Breve revisão da literatura}

Da literatura é possível extrair uma enorme quantidade de trabalhos que de uma forma direta ou indireta tem relação com o assunto discutido neste artigo; vide por exemplo Mula et al. (2006) que traz em seu conteúdo uma revisão de modelos para planejamento da produção sob incerteza. Independentemente de qual seja o grau dessa relação, todos têm em comum o fato de discutirem uma formulação para modelagem e geração de planos de produção, que levem em conta a natureza dinâmica estocástica do sistema produtivo sujeito a restrições físicas. Dentre esses trabalhos, podem-se destacar autores como Kleindorfer et al. (1975), Pekelman e Rausser (1978), Bitran e Yanasse, (1984), Feiring e Sastri (1990) e Yildirin et al. (2005), os quais têm desenvolvido heurísticas para lidar com problemas de planejamento da produção formulados com base em modelos seqüenciais, estocásticos, e com restrições probabilísticas nas variáveis de decisão. As heurísticas propostas por estes autores são baseadas em aproximações realizadas nas funções e variáveis aleatórias do modelo estocástico original, tornando-o assim um modelo equivalente mais simples de ser resolvido numericamente.

A seguir, apresenta-se um breve resumo de alguns trabalhos, publicados na vasta literatura existente sobre o tema e que estão próximos do contexto deste trabalho:

a) Kleindorfer et al. (1975) discutem e demonstram que é possível desenvolver planos de produção aplicando abordagens da teoria de controle ótimo estocástico em problemas de planejamento agregado da produção. Eles discutem o uso de heurísticas subótimas como um caminho de simplificar o problema estocástico seqüencial, de modo a viabilizá-lo computacionalmente;

b) Pekelman e Rausser (1978) discutem a importância do uso da informação no processo de tomada de decisão, e apresentam um conjunto de heurísticas que tomam vantagem da informação disponível do sistema para gerar políticas ótimas de produção para problemas de planejamento de produção estocásticos seqüenciais;

c) Bitran e Yanasse (1984) consideram um problema de planejamento da produção estocástico com uma condição de nível de serviço. Os autores propõem formulações determinísticas equivalentes para o modelo e determinam limites de erros entre as soluções estáticas (não seqüenciais) e as soluções exatas geradas;

d) Feiring e Sastri (1990) analisam um plano de produção com estratégias de horizonte deslizante e níveis de satisfação do cliente fixados pelos gerentes. As restrições probabilísticas do modelo são transformadas em restrições determinísticas, assumindo a hipótese da demanda ser normalmente distribuída;

e) Rao e Schneller IV (1990), no mesmo caminho considerado por Bitran and Yanasse (1984), identificam uma família de aproximações que podem ser usadas para simplificar um problema estocástico, porém, não sequiencial, adotado pelos autores para representar um problema de planejamento agregado de longo prazo;

f) Shen (1994) analisou três métodos de controle ótimo estocástico. Esses métodos foram aplicados na solução de um problema determinístico equivalente formulado com base no modelo quadrático clássico HMMS (HOLT et al., 1960). Dois dos métodos estudados incorporam medidas dos níveis de estoque observadas diretamente do sistema de produção (via algum esquema de realimentação de informação) para desenvolver a política subótima de produção;

g) Hackman et al. (2002) consideram um problema de planejamento da produção estocástico seqüencial cujo o interesse é determinar quando disponibilizar produtos acabados para atender a demanda prevista. $\mathrm{Na}$ solução, os autores consideram níveis de qualidade de serviço definidos a partir das restrições de chance da variável de estoque e adotam um esquema de horizonte deslizante para promover ajustes periódicos na política de produção;

h) Yildirin et al. (2005) analisam um problema conjunto de suprimento e planejamento da produção seqüencial estocástico com restrições de chance, que são transformadas em indicadores de nível de serviço ao cliente. Uma abordagem alternativa de solução do problema, baseada no procedimento de horizonte deslizante, é proposta pelos autores;

i) Rantala e Koivisto (2005) apresentam um algoritmo para geração de um plano agregado, a partir de um problema de planejamento da produção estocástico seqüencial, que leva em conta requisitos de qualidade de serviço; e

j) Levi et al. (2005) discutem, na mesma linha dos autores anteriores, modelos e técnicas aproximativas para lidar com problemas de planejamento da produção, procurando melhorar a solução gerada a partir de revisões periódicas.

Analisando os trabalhos apresentados acima é possível destacar alguns procedimentos adotados pelos autores na solução de problemas estocásticos seqüenciais, a saber: a) simplificação do problema estocástico, para torná-lo de mais simples solução; b) utilização de mecanismos de realimentação para ajustar a variável de decisão em 
relação ao estado do sistema; c) adoção de esquemas do tipo horizonte deslizante, para atualização da solução do problema com respeito à disponibilidade de informação no período de estudo; e d) fixação de critérios para melhoria do nível de satisfação do cliente.

Neste artigo, a principal contribuição esperada está associada ao uso simultâneo dos diferentes procedimentos da literatura, comentados sucintamente acima, na solução de problemas de planejamento estocásticos seqüenciais, com ênfase aqui no problema de geração de planos de produção. Deste modo, adota-se um esquema de aproximações matemáticas para transformar o problema estocástico original em um equivalente determinístico de mais simples solução. Algumas características interessantes resultantes deste processo de transformação são as de preservar propriedades e singularidades do problema original, como: a) a convexidade do critério a se otimizar; b) a linearidade do sistema e das restrições probabilísticas associadas, conservando particularidades estatísticas inerentes às variáveis de decisão; e c) a garantia do nível de serviço quanto ao atendimento do cliente (medido, por exemplo, pela capacidade de pronta entrega). Esta medida de atendimento está presente no modelo estocástico original na forma da restrição probabilística relacionada aos níveis de estoques, e que é mantida explicitamente na formulação do modelo determinístico equivalente, como veremos à frente. Além disto, é incluído neste modelo um mecanismo de realimentação para ajustes no tempo da variável de produção. $\mathrm{Na}$ solução proposta considera-se uma heurística clássica da teoria de controle para solução do problema determinístico com esquema de horizonte deslizante.

\subsection{Desdobramentos do trabalho}

O artigo está distribuído como se segue: a seção 2 considera a modelagem do problema de geração de planos de produção, através do uso do procedimento OLFC. Para este fim, é introduzido o problema de planejamento da produção seqüencial estocástico com restrições de chance nas variáveis de decisão. Como resultado, um problema equivalente determinístico baseado em aproximações estatísticas aplicadas ao problema original é apresentado. Com base nesse modelo equivalente, aplica-se o algoritmo de programação dinâmica levando em conta as etapas do procedimento OLFC. Em seguida, a seção 3 apresenta um exemplo ilustrativo de desenvolvimento de um plano de produção revisado usando a metodologia considerada na seção 2. Com este exemplo pretende-se responder algumas questões relacionadas à qualidade do serviço e à quase-adaptabilidade da heurística OLFC. Esta última questão é analisada comparando-se a solução revisada obtida via OLFC com as soluções malha-fechada (obtida pelo algoritmo de programação dinâmica estocástica) e malha-aberta, resultante do problema da média (também chamada de solução estática ou não revisada no tempo). Por fim, analisa-se o comportamento das políticas geradas pelos três procedimentos quando sujeitos a uma demanda projetada a partir de um modelo de previsão.

\section{Plano de produção via OLFC}

A geração de uma solução ótima verdadeira (i.e., uma solução global) para um problema que considera conjuntamente componentes dinâmicos, aleatórios e restritivos do processo produtivo, não é uma atividade trivial, mesmo para os mais versados nas teorias de otimização e controle ótimo. Assim sendo, grande atenção tem sido dada às abordagens ditas quase ótimas ou subótimas (BERTESEKAS, 2000). Estas técnicas seqüenciais subótimas são abordagens muito interessantes, na medida em que a maioria delas permite manter atualizações no tempo das variáveis de decisão (KLEINDORFER et al., 1975). Dentre as abordagens que adotam esquema de realimentação, escolheu-se para aplicação neste trabalho, o procedimento open-loop feedback control (OLFC) (SILVA FILHO, 2000; BERTESEKAS, 2000). A vantagem deste procedimento é que ele leva em conta explicitamente as incertezas relacionadas com as variáveis de decisão do problema original quando da obtenção da solução aproximada (i.e., solução subótima). Além disto, informações correntes sobre os níveis de estoque e de demanda são incorporadas periodicamente à solução do problema. É interessante notar que esta característica de atualização da informação torna a abordagem OLFC uma heurística dita do tipo adaptativa (BERTESEKAS, 2000). Isto significa que a solução fornecida por este procedimento é melhor do que aqueles procedimentos subótimos que não levam em conta informações parciais sobre o sistema produtivo. Tais soluções são conhecidas como soluções estáticas no tempo e advêm de problemas não seqüenciais (RAO; SCHNELLER IV, 1990). De fato, nas soluções temporalmente estáticas, nenhum tipo de atualização é considerado e o problema é resolvido "sempre" uma única vez, tendo como única informação o nível de estoque medido no período inicial do horizonte de planejamento.

Antes de serem discutidos os passos do procedimento OLFC, apresentam-se a seguir aspectos da formulação do problema de planejamento da produção que permitirá a geração de planos de produção seqüenciais.

\subsection{Problema de planejamento da produção}

Uma política ótima de produção $\left\{\mathrm{u}_{\mathrm{k}}^{*}=\mu\left(\mathrm{x}_{\mathrm{k}}\right) ; \mathrm{k}=\right.$ $0,1, \ldots, \mathbf{N}-1\}$ deve ser obtida como solução do seguinte problema de planejamento da produção seqüencial estocástico: 


$$
\begin{aligned}
& \underset{\mathrm{u}_{\mathrm{k}}}{\operatorname{Min}} \underset{\mathrm{d}_{\mathrm{k}}}{\mathrm{E}}\left\{\mathrm{f}\left(\mathrm{x}_{\mathrm{N}}\right)+\sum_{\mathrm{k}=0}^{\mathrm{N}-1} \mathrm{f}\left(\mathrm{x}_{\mathrm{k}}, \mathrm{u}_{\mathrm{k}}, \mathrm{d}_{\mathrm{k}}\right)\right\} \\
& \quad \mathrm{x}_{\mathrm{k}+1}=\mathrm{x}_{\mathrm{k}}+\mathrm{u}_{\mathrm{k}}-\mathrm{d}_{\mathrm{k}} ; \operatorname{com} \mathrm{E}\left\{\mathrm{x}_{0}\right\} \text { dado } \\
& \text { Prob. }\left(\mathrm{x}_{\mathrm{k}+1} \in X=\left\{\underline{\mathrm{x}} \leq \mathrm{x}_{\mathrm{k}+1} \leq \overline{\mathrm{x}}\right\}\right) \geq 2 \cdot \alpha-1 \\
& \text { Prob. }\left(\mathrm{u}_{\mathrm{k}} \in \mathrm{U}=\left\{\underline{\mathrm{u}} \leq \mathrm{u}_{\mathrm{k}} \leq \overline{\mathrm{u}}\right\}\right) \geq 2 \cdot \beta-1
\end{aligned}
$$

em que $E\{\cdot\}$ é o operador esperança matemática. As variáveis $\mathrm{x}_{\mathrm{k}}, \mathrm{u}_{\mathrm{k}}$, e $\mathrm{d}_{\mathrm{k}}$ denotam níveis de estoque, produção e demanda para cada período de tempo $\mathrm{k} \in[0, \mathrm{~N}-1] \mathrm{e}$ os pares $(\underline{\mathrm{x}}, \overline{\mathrm{x}}$,$) e (\underline{\mathrm{u}}, \overline{\mathrm{u}})$ representam respectivamente os limitantes inferiores e superiores dos conjuntos $\mathrm{X}$ e $\mathrm{U}$, relacionados com as variáveis de estoque e produção. É interessante observar que os limitantes inferiores $\underline{x}$ e $\underline{u}$ denotam respectivamente, em termos práticos, os níveis aceitáveis de estoque de segurança e de ociosidade na capacidade de produção permitidos pela empresa ao longo do horizonte de planejamento. Do mesmo modo, $\overline{\mathrm{x}}$ e $\overline{\mathrm{u}}$ denotam os níveis máximos operacionais de armazenagem e de capacidade de produção, respectivamente. A função $\mathrm{f}(\cdot)$ representa o custo total de produção do problema, que se deseja minimizar. Os parâmetros $\alpha$ e $\beta \in[1 / 2,1)$ são índices probabilísticos que devem ser definidos pelo usuário e cujas características de interesse serão discutidas à frente. Por fim, os níveis de estoque inicial $x_{0}$ e final $x_{N}$ devem ser estimados a priori pelo responsável pela elaboração da política de decisão.

\subsubsection{Características do modelo de balanço de estoques e restrições}

A natureza aleatória do problema (1)-(4) tem origem no fato do sistema linear de balanço de estoque ser um processo estocástico. Este processo é dependente do comportamento da demanda (i.e., dos níveis agregados de venda de produtos similares) que no longo prazo não é conhecida exatamente. Neste estudo é considerado que a demanda segue um padrão estacionário caracterizado por uma função distribuição normal de probabilidade. Esta função é conhecida ao longo do tempo através dos seus dois primeiros momentos estatísticos dados, respectivamente, pela média $\hat{d}_{\mathrm{k}}$ e variância finita $\sigma_{\mathrm{d}}^{2} \geq 0$. É importante destacar que num ambiente de produção do tipo orientado a estoque (HIGGINS et al.,1996), onde a evolução temporal da demanda usualmente segue um padrão estacionário, a distribuição normal de probabilidade é uma alternativa viável para representar estatisticamente as flutuações de demanda (GRAVES, 1999).

Como consequiência da distribuição normal de probabilidade da variável de demanda, o sistema linear de balanço de estoque, descrito em (2), pode ser completamente caracterizado por um processo estocástico dito
Gaussiano (BRYSON; HO, 1975). Assim, o nível de estoque é uma variável aleatória completamente caracterizada pela evolução da média $\hat{x}_{\mathrm{k}}$ e da variância $\mathrm{V}_{\mathrm{x}}^{\mathrm{k}}$. Uma conseqüência imediata da natureza aleatória do processo de estoque é a impossibilidade de se garantir com certeza que os níveis de estoque $\mathrm{x}_{\mathrm{k}}$ respeitarão os limites físicos do espaço de armazenagem, denotado em (3) por $\mathrm{X}$. Assim, visando permitir que essa restrição permaneça explicitamente na formulação do problema estocástico, ela é considerada uma restrição de chance (VADJA, 1972), ou seja, uma restrição garantida em probabilidade, como proposto em (3).

De modo análogo ao parágrafo discutido acima, a variável de produção está restrita a tomar valores de um espaço probabilístico gerado pela restrição (4). A razão disto é que a variável de produção $u_{k}$ é função no tempo dos níveis de estoques $\mathrm{x}_{\mathrm{k}}$, que são variáveis aleatórias. Isto significa que as medidas dos níveis de estoques são usadas para ajustar a política de produção a cada período de tempo k. Matematicamente, isto pode ser expresso como $u_{k}=\mu\left(x_{k}\right)$, em que $\mu$ é uma função que mapeia o espaço da variável $\mathrm{x}_{\mathrm{k}}$ no espaço da variável $\mathrm{u}_{\mathrm{k}}$. Decorre deste fato que $\mathrm{u}_{\mathrm{k}}$ é também uma variável aleatória e para garantir que $u_{k} \in U$, sem risco de violação, é necessário também considerá-la em probabilidade.

Apenas a título de curiosidade, é interessante observar que a gerência pode usar o nível de estoque de segurança $\underline{x}$, associado com diferentes valores de $\alpha$, para criar cenários de produção em que se busca a eficiência na qualidade de serviço (HACKMAN et al., 2002). Isto explica o motivo pelo qual o índice probabilístico $\alpha$ é muitas vezes conhecido como uma medida quantitativa do nível de serviço ao cliente (i.e., índice que determina qual o grau de satisfação do cliente que a gerência pretende desenvolver). Quanto maior for o valor escolhido para o índice $\alpha \in$ $[1 / 2,1]$, maior serão as chances de satisfazer o cliente. Por exemplo, se for fixado $\alpha=0,95$ significa que haverá 95\% de chances de que o produto será entregue ao cliente na data combinada.

\subsubsection{Simplificando o modelo estocástico}

Para facilitar o processo de transformação do problema estocástico (1)-(4) em um problema determinístico equivalente de mais simples solução, consideram-se, sem prejuízo à generalidade, os seguintes aspectos:

$\mathbf{1}^{\mathbf{0}}$ Aspecto: A relação entre as variáveis de produção e de estoque, expressa genericamente como $u_{k}=\mu\left(x_{k}\right)$, é agora definida por uma constante $\mathrm{G}$, ou seja, $\mu=-\mathrm{G} \cdot \mathrm{x}_{\mathrm{k}}$, que denota um ganho linear que pondera a relação entre a quantidade produzida $u_{k}$ e a quantidade armazenada $\mathrm{x}_{\mathrm{k}}$ para cada período $\mathrm{k}$ do horizonte de planejamento. $\mathrm{O}$ cálculo deste ganho pode ser feito, por exemplo, usando algum esquema de ajuste de controladores adaptativos, como os do tipo proporcional (McMILLAN, 1983). 
Um caminho alternativo para determinar o ganho $\mathrm{G}$ consiste em resolver um problema de variância mínima (ASTRÖM, 1970), ou seja:

$$
\operatorname{Min}_{\mathrm{G}}\left\{\mathrm{V}_{\mathrm{x}}^{\mathrm{k}+1}+\eta \cdot \mathrm{V}_{\mathrm{u}}^{\mathrm{k}}\right\}
$$

cuja solução permite reduzir simultaneamente as amplitudes das variâncias das variáveis de estoque $\mathrm{V}_{\mathrm{x}}^{\mathrm{k}} \mathrm{e}$ de produção $\mathrm{V}_{\mathrm{u}}^{\mathrm{k}}$.

O problema de variância mínima, como formulado em (5), está fundamentado no fato de que a incerteza sobre o comportamento futuro da demanda $\mathrm{d}_{\mathrm{k}}$ provoca um aumento gradativo nas amplitudes das variâncias de estoque e de produção com o avançar dos períodos. Este efeito está ilustrado na Figura 2, na qual se observa a evolução no tempo da função distribuição de probabilidade da variável de estoque sem qualquer tipo de interferência ou ajuste. Devido à linearidade da relação $\mathrm{u}_{\mathrm{k}}=-\mathrm{G} \cdot \mathrm{X}_{\mathrm{k}}$, a variável de produção terá uma distribuição de probabilidade similar a da variável de estoque, e o crescimento do segundo momento estatístico é semelhante ao comportamento ilustrado na Figura 2.

Assim sendo, a minimização simultânea do crescimento dessas variâncias permite obter um plano de produção mais confiável e "estável", pois a variabilidade das variáveis de estoque e produção estão sob controle, ou seja, ajustadas continuamente ao longo do tempo. É importante frisar que esta estabilidade é obtida através da seleção do parâmetro $\eta$ que funciona como um "ajuste de equilíbrio", em que as amplitudes das variâncias de estado e produção são mantidas balanceadas. Com isto, pode-se garantir a redução dos níveis de variabilidade das variáveis de estoque e produção ao longo dos períodos.

A solução do problema (5) gera um ganho ótimo dado por $G_{\eta}^{*}=1 /(1+\eta)$, cujo o parâmetro de ponderação $\eta$ pode ser calculado de diferentes maneiras, uma das mais simples, descrita detalhadamente em Silva Filho e Ventura (1999), relaciona os limites de estoque e produção das restrições (3) e (4) para calcular $\eta$, como segue:

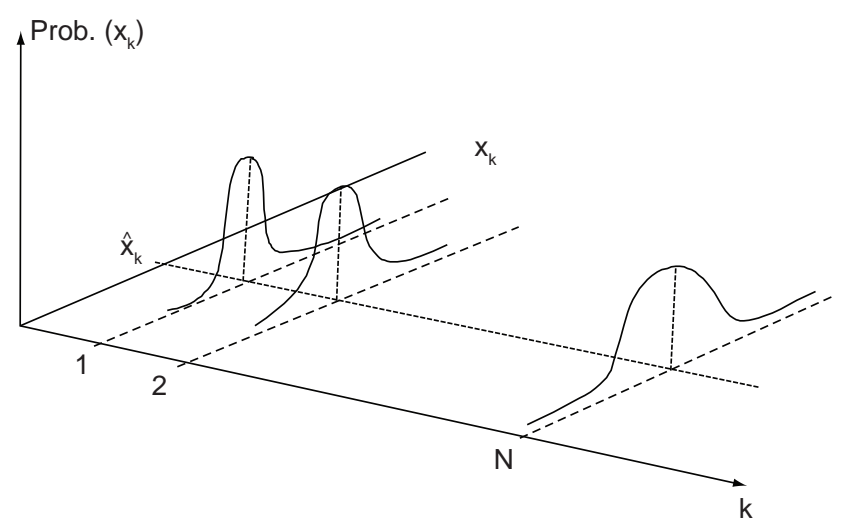

Figura 2. Evolução da distribuição de probabilidade da variável de estoque.

$$
\eta=(\overline{\mathrm{x}}-\underline{\mathrm{x}})^{2} /(\overline{\mathrm{u}}-\underline{\mathrm{u}})^{2}
$$

$\mathbf{2}^{\mathbf{0}}$ Aspecto: O índice $\beta$ será fixado aqui em $50 \%$ (i.e., $\beta=0,50)$. Com isto o espaço de restrição da variável de produção $\mathrm{u}_{\mathrm{k}}$ é exatamente igual ao seu espaço físico. Isto só é possível de ser realizado devido ao padrão de demanda ser do tipo estacionário (vide seção 2.1.1). Somando-se a isto, o fato das variâncias de estoque e produção estarem sendo minimizadas no tempo pela formulação dada em (5), é possível considerar que a capacidade de produção terá grandes chances de não sofrer violações ao longo dos períodos futuros de tempo; e por fim,

$3^{\circ}$ Aspecto: Como discutido na seção 2.1.1, a demanda é definida por uma seqüência de variáveis aleatórias independentes $\left\{\mathrm{d}_{\mathrm{k}}, \mathrm{k}=0,1, \ldots, \mathrm{N}\right\}$ que são aproximadas por uma função distribuição de probabilidade normal, com média conhecida $\hat{\mathrm{d}}_{\mathrm{k}}$ e variância finita no tempo $\sigma_{\mathrm{d}}^{2}>0, \forall \mathrm{k}$. Com base nesta suposição de normalidade estocástica, o valor residual da variável de demanda, definida como $\varepsilon_{\mathrm{k}}=\left(\mathrm{d}_{\mathrm{k}}-\hat{\mathrm{d}}_{\mathrm{k}}\right) / \sigma_{\mathrm{d}}$, terá uma função distribuição de probabilidade normal descrita por $\Phi_{\varepsilon_{\mathrm{k}}}(\gamma)=$ Prob. $\left(\varepsilon_{\mathrm{k}} \leq \gamma\right) \geq \alpha$, com $\gamma$ sendo um valor que delimita superiormente a existência da variável aleatória $\varepsilon_{\mathrm{k}}$. Neste caso, o índice de probabilidade $\alpha$, fixado a priori pelo decisor, representa um indicador probabilístico de validade desta relação. Baseado na teoria das probabilidades, o valor de $\gamma$ pode ser estatisticamente determinado por $\gamma \geq \Phi_{\varepsilon_{\mathrm{k}}}^{-1}(\alpha) \operatorname{com} \Phi_{\varepsilon_{\mathrm{k}}}^{-1}$ denotando a função inversa da distribuição de probabilidade.

Segundo Graves (1999), a hipótese de distribuição de probabilidade normal para representar flutuações estacionárias de demanda tem forte apelo nas práticas de planejamento da produção. Outra importante justificativa para uso desta hipótese estatística é a lei dos grandes números, utilizada para ambientes dinâmicos a tempos discretos, cuja a componente resultante da soma de diferentes tipos de distribuições de probabilidade pode ser aproximada por uma função distribuição normal (PAPOULIS, 1991). A vantagem de usar distribuição normal está no fato dela ser completamente definida por apenas dois momentos estatísticos, a saber: a média e a variância do processo normal. Assim, usando esta distribuição, algumas simplificações podem ser imediatamente realizadas no problema (1)-(4), como apresentadas a seguir:

Função objetivo: o custo esperado de produção pode ser calculado para cada período de tempo k, como segue:

$$
\begin{gathered}
\underset{x_{0}, d_{k}}{E}\left\{\sum_{k=0}^{N-1} f\left(x_{k}, u_{k}, d_{k}\right)\right\}= \\
\sum_{k=0}^{N-1} \underset{x_{0}, d_{k}}{E}\left\{f\left(x_{k}, u_{k}, d_{k}\right)\right\}=\sum_{k=0}^{N-1} F\left(x_{k}\right)
\end{gathered}
$$


em que $\mathrm{F}\left(\hat{\mathrm{x}}_{\mathrm{k}}\right)=\underset{\mathrm{x}_{0}, \mathrm{~d}_{\mathrm{k}}}{\mathrm{E}}\left\{\mathrm{f}\left(\mathrm{x}_{\mathrm{k}}, \mathrm{u}_{\mathrm{k}}, \mathrm{d}_{\mathrm{k}}\right)\right\}=\int_{\Re} \mathrm{f}\left(\mathrm{x}_{\mathrm{k}}, \mathrm{G} \cdot \mathrm{x}_{\mathrm{k}}, \varepsilon_{\mathrm{k}}\right) \mathrm{d} \Phi_{\varepsilon_{\mathrm{k}}}$, com $\hat{x}_{\mathrm{k}}=\mathrm{E}\left\{\mathrm{x}_{\mathrm{k}}\right\}$. Note que a função $\mathrm{F}($.) deve ser calculada numericamente para cada período $\mathrm{k}$. Caso $\mathrm{f}($.) seja convexa, a função $\mathrm{F}($.) resultante manterá essa mesma propriedade (LASSERE et al., 1984).

Restrições probabilísticas: as restrições (3) e (4) podem também ser precisamente calculadas para cada período de tempo k. Assim, considerando que o sistema de balanço de estoque (2) é um processo estocástico normalmente distribuído, é possível transformar as restrições probabilísticas em restrições determinísticas equivalentes que dependem diretamente de seus dois primeiros momentos estatísticos, ou seja, da evolução da média e da variância. Assim, a conversão das restrições probabilísticas segue basicamente as seguintes etapas:

Etapa (a): inicialmente considere a restrição de chance dada em (3). É possível mostrar que Prob. $\left(\underline{\mathrm{x}} \leq \mathrm{x}_{\mathrm{k}+1} \leq \overline{\mathrm{x}}\right) \geq 2 . \alpha-1$ pode ser aproximado por Prob. $\left(\mathrm{x}_{\mathrm{k}+1} \leq \overline{\mathrm{x}}\right)+$ Prob. $\left(\mathrm{x}_{\mathrm{k}+1} \geq \underline{\mathrm{x}}\right) \geq 2$. $\alpha$, em que $\alpha \in[1 / 2$, 1) (SILVA FILHO; GEROMEL, 1990). Com base nisto, e considerando que $\mathrm{d}_{\mathrm{k}}=\hat{\mathrm{d}}_{\mathrm{k}}+\varepsilon_{\mathrm{k}} \cdot \sigma_{\mathrm{d}}$, em que $\hat{\mathrm{d}}_{\mathrm{k}}=\mathrm{E}\left\{\mathrm{d}_{\mathrm{k}}\right\}$ e $\sigma_{\mathrm{d}}^{2}=\mathrm{E}\left\{\mathrm{d}_{\mathrm{k}}^{2}\right\}$, é possível reescrever (3) do seguinte modo:

$$
\begin{gathered}
\operatorname{Prob} .\left(\varepsilon_{\mathrm{k}} \geq \frac{\mathrm{x}_{\mathrm{k}}+\mathrm{u}_{\mathrm{k}}-\overline{\mathrm{x}}-\hat{\mathrm{d}_{\mathrm{k}}}}{\sigma_{\mathrm{d}}}\right)+ \\
\text { Prob. }\left(\varepsilon_{\mathrm{k}} \leq \frac{\mathrm{x}_{\mathrm{k}}+\mathrm{u}_{\mathrm{k}}-\underline{\mathrm{x}}-\hat{\mathrm{d}}_{\mathrm{k}}}{\sigma_{\mathrm{d}}}\right) \geq 2 \cdot \alpha
\end{gathered}
$$

Após algumas manipulações algébricas realizadas em (8) e considerando que $u_{k}=-G_{\eta} \cdot x_{k}$, segue então que (8) é equivalente a:

$$
\begin{aligned}
& \mathrm{u}_{\mathrm{k}} \geq \frac{1}{1-\mathrm{G}_{\eta}} \cdot\left(\underline{\mathrm{x}}+\hat{\mathrm{d}}_{\mathrm{k}}+\sigma_{\mathrm{d}} \cdot \Phi_{\varepsilon_{\mathrm{k}}}^{-1}(\alpha)\right)=\underline{\mathrm{u}}_{\mathrm{G}, \alpha}^{\mathrm{k}} \\
& \mathrm{u}_{\mathrm{k}} \leq \frac{1}{1-\mathrm{G}_{\eta}} \cdot\left(\overline{\mathrm{x}}+\hat{\mathrm{d}}_{\mathrm{k}}-\sigma_{\mathrm{d}} \cdot \Phi_{\varepsilon_{\mathrm{k}}}^{-1}(\alpha)\right)=\overline{\mathrm{u}}_{\mathrm{G}, \alpha}^{\mathrm{k}}
\end{aligned}
$$

em que $0 \leq \mathrm{G}_{\eta}<1$. Esse ganho pode ser calculado através de métodos de ajustes via simulação ou pelo procedimento de Variância Mínima, como comentado previamente.

Etapa (b): A partir da desigualdade (9) e do conjunto $\mathrm{U} \in[\underline{\mathrm{u}}, \overline{\mathrm{u}}]$ é possível definir um subconjunto $\Omega_{\mathrm{u}}\left(\mathrm{x}_{\mathrm{k}}, \alpha\right)$ que contém somente as políticas de produção factíveis do problema. Este subconjunto é função do nível de estoque $x_{k}$ e do índice de satisfação do cliente $\alpha$. Os limites mínimos e máximos são dados para cada período de tempo k como segue:

$$
\begin{gathered}
\underline{\mathrm{u}}\left(\mathrm{x}_{\mathrm{k}}, \alpha\right)= \\
\operatorname{Max}\left[\underline{\mathrm{u}}, \underline{\mathrm{u}}_{\mathrm{G}, \alpha}^{\mathrm{k}}\right] \text { e } \overline{\mathrm{u}}\left(\mathrm{x}_{\mathrm{k}}, \alpha\right)=\operatorname{Min}\left[\overline{\mathrm{u}}, \overline{\mathrm{u}}_{\mathrm{G}, \alpha}^{\mathrm{k}}\right]
\end{gathered}
$$

É importante notar, que tendo sido calculado o valor do ganho de realimentação $\mathrm{G}$ e fixados os valores do índice probabilístico $\alpha$, os limites físicos estabelecidos por (10) ficam prontamente determinados e, por conseqüência, o espaço restritivo de produção $\Omega_{\mathrm{u}}\left(\mathrm{x}_{\mathrm{k}}, \alpha\right)$ plenamente determinado. Este espaço apresenta a seguinte propriedade:

Propriedade: Sejam $\alpha_{1}$ e $\alpha_{2}$ índices de probabilidade, tais que $\alpha_{2} \geq \alpha_{1}$, segue então que $\Omega_{\mathrm{u}}\left(\mathrm{x}_{\mathrm{k}}, \alpha_{2}\right) \subseteq \Omega_{\mathrm{u}}\left(\mathrm{x}_{\mathrm{k}}, \alpha_{1}\right)$, $\forall \mathrm{k}$.

A propriedade acima garante em probabilidade a factibilidade de solução do problema determinístico equivalente. Para verificar a validade desta propriedade assume-se uma condição contraditória em que $\Omega_{\mathrm{u}}\left(\mathrm{x}_{\mathrm{k}}\right.$, $\left.\alpha_{2}\right) \supset \Omega_{\mathrm{u}}\left(\mathrm{x}_{\mathrm{k}}, \alpha_{1}\right)$, para qualquer período $\mathrm{k} \operatorname{com} \alpha_{2} \geq \alpha_{1}$. Sob tal condição tem-se que $\underline{u}_{\mathrm{G}, \alpha_{2}}^{\mathrm{k}}>\underline{\mathrm{u}}_{\mathrm{G}, \alpha_{1}}^{\mathrm{k}}$ e/ou $\overline{\mathrm{u}}_{\mathrm{G}, \alpha_{2}}^{\mathrm{k}}>\overline{\mathrm{u}}_{\mathrm{G}, \alpha_{1}}^{\mathrm{k}}$. Como conseqüência, manipulando-se as condições dadas em (9) chega-se a seguinte condição: $\Phi_{\varepsilon}^{-1}\left(\alpha_{2}\right)<\Phi_{\varepsilon}^{-1}\left(\alpha_{1}\right)$ a qual não é válida do ponto de vista probabilístico. Com isto conclui-se que sempre $\Omega_{\mathrm{u}}\left(\mathrm{x}_{\mathrm{k}}, \alpha_{2}\right) \subseteq \Omega_{\mathrm{u}}\left(\mathrm{x}_{\mathrm{k}}, \alpha_{1}\right)$, para qualquer $\alpha_{2} \geq \alpha_{1}$.

A propriedade acima mostra que o espaço gerado $\Omega_{\mathrm{u}}\left(\mathrm{x}_{\mathrm{k}}, \alpha\right)$ fica mais restrito (isto é, tecnicamente menor) à medida em que o administrador toma a decisão de aumentar sua expectativa de satisfazer o cliente (o que significa fixar $\alpha$ próximo de $100 \%$ ). Em outras palavras, quanto maior for o interesse da empresa em satisfazer o cliente, menor será a chance de se encontrar uma solução factível para o problema estocástico (1)-(4). De fato, podese mostrar que além da redução do espaço de factibilidade do problema (1)-(4), explicitada pela propriedade acima, existe também um aumento significativo nos custos de produção, devido ao aumento acumulado dos níveis de estoque, visando atender a demanda projetada para os períodos futuros.

\subsection{O procedimento OLFC}

É importante destacar inicialmente que o problema (1)-(4) pertence à classe de problemas de controle ótimo estocástico sob perfeita informação do estado (BERTESEKAS, 2000). Isto significa que o nível real (ou seja, verdadeiro) de estoque do sistema pode ser medido exatamente no início de cada novo período de tempo $\mathrm{k}$. Esta característica do problema simplifica enormemente a aplicação da abordagem OLFC, uma vez que não há necessidade de uso de métodos de estimação de estados, baseados em mecanismos de filtragem (ASTRÖM, 1970), para identificar qual o nível de estoque corrente.

Como base no exposto acima se tem que, tão logo conhecido o nível exato de estoque para o período $\mathrm{k}$, é possível determinar a quantidade ótima de produtos a serem produzidos via procedimento OLFC, através dos seguintes passos:

Passo 1. A cada novo período de tempo, isto é, $\mathrm{t}=\mathrm{k}$, em que $\mathrm{k} \in[0, \mathrm{~N}-1]$, o administrador observa a posição 
exata dos níveis de estoque de cada produto. Isto é, ele mede precisamente qual é a quantidade real armazenada de cada um dos produtos, ou seja, $x_{t}=x_{k}$.

Passo 2. Tendo conhecido o nível de estoque $x_{t}, o$ administrador poderá determinar uma política ótima de produção $\left\{\mathrm{u}_{\mathrm{k}}, \mathrm{u}_{\mathrm{k}+1}, \ldots \mathrm{u}_{\mathrm{N}-1}\right\}$ para cada produto, que é a solução do seguinte problema equivalente:

$$
\begin{aligned}
& \operatorname{Min}_{\mathrm{u}_{\mathrm{k}}}\left\{\mathrm{F}\left(\hat{\mathrm{x}}_{\mathrm{N}}\right)+\sum_{\mathrm{t}=\mathrm{k}}^{\mathrm{N}-1} \mathrm{~F}\left(\hat{\mathrm{x}}_{\mathrm{k}}\right)\right\} \\
& \text { s.a. } \\
& \quad \hat{\mathrm{x}}_{\mathrm{t}+1}=\hat{\mathrm{x}}_{\mathrm{t}}+\mathrm{u}_{\mathrm{t}}-\hat{\mathrm{d}}_{\mathrm{t}} ; \quad \hat{\mathrm{x}}_{\mathrm{t}}=\mathrm{x}_{\mathrm{k}} \text { observado } \\
& \quad \mathrm{u}_{\mathrm{t}} \in \Omega_{\mathrm{u}}\left(\mathrm{x}_{\mathrm{t}}, \alpha\right) \\
& \mathrm{t}=\mathrm{k}, \mathrm{k}+1, \ldots, N-1 \text { e } \mathrm{k} \in[0, N-1]
\end{aligned}
$$

em que F(.) e $\Omega_{u}$ (.) são precisamente calculados, desde que o nível de estoque de cada produto seja precisamente observado para cada período $\mathrm{k} ; \hat{\mathrm{x}}_{\mathrm{t}}=\mathrm{E}\left\{\mathrm{x}_{\mathrm{t}}\right\}$ denota o valor esperado da variável de estoque para o período $\mathrm{k}$. É importante observar que, uma vez calculado $\mathrm{F}\left(\right.$.) e $\Omega_{\mathrm{u}}($.), o problema (11) torna-se um problema essencialmente determinístico e sua solução ótima depende apenas do valor de estoque observado no início de cada período k. Além disto, a solução gerada por (11) não considera nenhuma informação atualizada sobre os níveis de estoque que possa estar disponível para períodos $\mathrm{t}>\mathrm{k}$. Em outras palavras, qualquer informação adicional projetada a respeito dos níveis de estoque nos períodos futuros do horizonte de planejamento é completamente ignorada pelo problema (11). Como consequiência, o plano de produção gerado por (11) é uma solução congelada no tempo, ou seja, é uma solução malha aberta, como costuma ser o jargão adotado por praticantes da teoria de controle (BERTESEKA, 2000).

Passo 3. somente a taxa ótima de produção $u_{t}^{*}$, calculada para o período $\mathrm{t}=\mathrm{k}$, a partir do problema (11), será efetivamente aplicada no processo hierárquico de decisão. Servindo assim como meta de produção estabelecida para o período k, como ilustrado pela Figura 1, na seção introdutória.

É importante destacar por fim, que estes três passos são repetidos continuamente ao longo dos períodos até que se tenha completado o horizonte de planejamento em estudo.

\subsection{Características do procedimento OLFC}

As principais características relacionadas com a abordagem OLFC são: a) ela requer a solução do problema (11) num total de $\mathrm{n}$ vezes. Isto significa que a cada nova medição do estado de balanço de estoque, o problema (11) deve ser resolvido segundo um padrão semelhante àquele conhecido na literatura como horizonte deslizante (PEREIRA; SOUSA, 1997; YILDIRIM et al., 2005); b) como observado nos passos 2 e 3 , para cada solução do problema (11), somente a política ótima de decisão $u_{t}^{*}$, calculada para o período $\mathrm{t}=\mathrm{k}$ é efetivamente utilizada como estratégia de decisão. Isto significa, que o resto da política ótima gerada, i.e., $\left\{\mathrm{u}_{\mathrm{k}+1}, \mathrm{u}_{\mathrm{k}+2}, \ldots, \mathrm{u}_{\mathrm{N}-1}\right\}$, é completamente descartada para efeito de aplicação na hierarquia de planejamento descrita pela Figura 1; c) a abordagem OLFC é de fácil implementação computacional, com aplicação a problemas de grande dimensão. Além disso, o problema (11) pode ser resolvido por qualquer técnica aplicável da teoria de controle ótimo e/ou da programação matemática (CHENG et al., 2004; SHEN, 1994; NECK, 1984); d) fixando $\mathrm{t}=\mathrm{k}=0$ e $\mathrm{d}_{\mathrm{k}}=\hat{\mathrm{d}}_{\mathrm{k}}$ com $\mathrm{V}_{\mathrm{d}}^{\mathrm{k}}=\sigma_{\mathrm{d}}^{2}=0, \forall \mathrm{k}$, o problema (11) torna-se um problema determinístico conhecido como problema da média (SILVA FILHO; VENTURA, 1999), cuja a solução é malha-aberta (BERTESEKA, 2000); e e) uma vez que o procedimento OLFC incorpora informações correntes sobre os níveis de estoque do sistema, disponíveis a cada novo período $\mathrm{k}$, ela é dita ser quase-adaptativa. Isto significa que tal procedimento fornece uma solução para o problema (11) que é melhor do que a solução gerada por outros procedimentos baseados em esquemas ditos malha-aberta.

\section{Exemplo}

O objetivo desta seção é analisar a aplicação do procedimento OLFC em um problema de planejamento da produção seqüencial, estocástico e com restrições de chance. Para efeito deste estudo, as seguintes questões são propostas, a saber: a) qual a influência do índice probabilístico $\alpha$ na política ótima fornecida pela heurística OLFC? b) é possível confirmar numericamente a natureza quase adaptativa da heurística OLFC? e c) qual o comportamento da política OLFC quando aplicada a um processo de balanço de estoque submetido a uma demanda projetada?

Estas três questões serão respondidas a partir da solução de um problema muito simples de planejamento agregado da produção, que é formulado a seguir.

$\mathrm{O}$ problema aqui proposto consiste em determinar um plano ótimo de produção para uma única família de produtos. A idéia é minimizar a esperança matemática da função quadrática $\mathrm{E}\left\{\mathrm{f}\left(\mathrm{x}_{\mathrm{k}}, \mathrm{u}_{\mathrm{k}}\right)\right\}=\mathrm{h} . \mathrm{E}\left\{\mathrm{x}_{\mathrm{k}}^{2}\right\}+\mathrm{c} .\left\{\mathrm{u}_{\mathrm{k}}^{2}\right\}$, satisfazendo, simultaneamente, restrições associadas ao balanço de estoque (i.e., $\mathrm{x}_{\mathrm{k}+1}=\mathrm{x}_{\mathrm{k}}+\mathrm{u}_{\mathrm{k}}-\mathrm{d}_{\mathrm{k}}$ ) e às capacidades de armazenagem do produto e de operação da produção, dadas respectivamente por Prob. $\left(\mathrm{x}_{\mathrm{k}+1} \in \mathrm{X}\right)$ $\geq 2 \cdot \alpha-1$ e Prob. $\left(u_{k} \in U\right) \geq 2 \cdot \beta-1$.

$\mathrm{O}$ fato do exemplo considerar um modelo constituído por uma única família de produtos torna possível a aplicação do algoritmo de Programação Dinâmica Estocástica (PDE). Este algoritmo permite que se produza uma solução ótima global para problemas de pequena 
dimensão (BERTESEKAS, 2000). Assim, aplicando o algoritmo PDE ao problema descrito acima, podese formular o segundo passo do procedimento OLFC, conforme discutido na subseção 2.2 , como segue: a cada novo período de tempo $\mathrm{t}=\mathrm{k}$ do horizonte de planejamento $\mathrm{N}$, o seguinte problema deve ser resolvido:

Dado

$$
\mathrm{J}_{\mathrm{N}}\left(\mathrm{x}_{\mathrm{N}}\right)=\psi(\mathrm{N})
$$

Determine

$$
\mathrm{J}_{\mathrm{t}}\left(\mathrm{x}_{\mathrm{t}}\right)=\operatorname{Min}_{\substack{\mathrm{u}_{\mathrm{t}} \in \Omega\left(\mathrm{x}_{\mathrm{t}}, \alpha\right) \\ \mathrm{x}_{\mathrm{t}+1}=\mathrm{x}_{\mathrm{t}}+\mathrm{u}_{\mathrm{t}}-\mathrm{d}_{\mathrm{t}} \\ \mathrm{t}=\mathrm{k}, \mathrm{k}+1, \ldots, \mathrm{N} \\ \mathrm{k}=0,1,2, \ldots, \mathrm{N}-1}}\left\{\psi(\mathrm{t})+\mathrm{J}_{\mathrm{t}+1}(\psi(\mathrm{t}+1))\right\}
$$

em que $\mathrm{J}(\cdot)$ denota o custo funcional do problema descrito em (12) e que segue a convenção da literatura, como apresentado em Bertesekas (2000). A função $\psi(\cdot)$ é descrita como segue:

$$
\begin{aligned}
\psi(t) & \left.=\underset{d_{t}}{E}\left\{f\left(x_{t}, u_{t}\right)\right)\right\}=h \cdot \underset{d}{\mathrm{E}}\left\{x_{t}^{2}\right\}+\underset{d}{c} \cdot \underset{d}{E}\left\{u_{t}^{2}\right\} \\
& =\left(h+c \cdot G^{2}\right) \cdot\left[(1-G)^{2 \cdot(t-k)} \cdot x_{k}^{2}+t \cdot \sigma_{d}^{2}-2 .\right. \\
& \left.(1-G)^{t-k} \cdot x_{k} \cdot \sum_{i=k}^{t} d_{i}\right] ; t=k, k+1, \cdots, N-1
\end{aligned}
$$

Com respeito à expressão do custo (13), é interessante notar que a variável $\mathrm{x}_{\mathrm{k}}$ representa o valor observado (ou medido) no início de cada novo período $\mathrm{t}=\mathrm{k}$, não sendo, portanto, uma variável aleatória. Outro aspecto considerado aqui é que os níveis de demanda $\left\{\mathrm{d}_{\mathrm{i}} ; \mathrm{i}=\mathrm{k}, \mathrm{k}+1, \ldots\right.$, $\mathrm{N}-1\}$ são considerados independentes e, portanto, qualquer correlação entre eles é nula para efeito de cálculo.

Como resultado da solução do problema (12), a taxa ótima de produção $u_{t}^{*}$ é obtida para aplicação no período $\mathrm{t}=\mathrm{k}$. Note que para o período $\mathrm{k}$, esta taxa de produção é usada como meta a ser considerada nas decisões tomadas pelos níveis subseqüentes da hierarquia, como ilustra a Figura 1 da seção introdutória.

Os dados do problema válidos para cada período mensal $\mathrm{k}$ do horizonte de planejamento $\mathrm{N}=12$ meses são descritos como seguem:

a) nível de estoque de segurança fixado pelo administrador $\underline{x}=4$ unidades e nível máximo de armazenagem da família $\bar{x}=19$ unidades;

b) capacidade mínima e máxima real adotada pela organização $\underline{u}=4$ e $\overline{\mathrm{u}}=9$ unidades;

c) a demanda é uma variável aleatória que segue um comportamento normal, cuja justificativa foi apre- sentada na subseção 2.1.2 e, portanto, tem sua natureza estocástica descrita completamente por um desvio-padrão finito $\sigma_{\mathrm{d}}=0,5$ e por uma flutuação média mensal $\hat{\mathrm{d}}_{\mathrm{k}}$ expressa na Tabela 1 ;

d) os custos de estoque e produção, em alguma unidade monetária, são dados respectivamente por $\mathrm{h}=2,0 \mathrm{e}$ $\mathrm{c}=1,0 ; \mathrm{e}$ (e) o estoque inicial é fixado em $\mathrm{x}_{0}=10$; e

e) a relação linear envolvendo a quantidade necessária para produzir a família de produtos e a quantidade a armazenar é balanceada pelo ganho linear $\mathrm{G}=1 /(1+\eta)$, com $\eta=(\overline{\mathrm{x}}-\underline{\mathrm{x}})^{2} /(\overline{\mathrm{u}}-\underline{\mathrm{u}})^{2}$, como descrito na seção 2.1.2. Para este estudo tem-se que: $\eta=9 \Rightarrow \mathrm{G}=0,10$.

\subsection{Resultados principais}

Com objetivo específico de responder a questão relacionada à influência do índice $\alpha$ sobre o plano de produção, serão analisados dois casos extremos com valores de $\alpha$ iguais a 0,5 e 0,95 . No primeiro há um risco de $50 \%$ da demanda não ser totalmente atendida até o final do horizonte de planejamento. Isto significa que, para algum período $\mathrm{k}$ deste horizonte, poderá ocorrer de não ser possível se obter uma taxa de produção factível, isto é, $\mathrm{u}_{\mathrm{k}} \notin \Omega\left(\mathrm{x}_{\mathrm{k}} ; \alpha=1 / 2\right)$. Essa situação pode ser observada em algum tipo de negócio em que haja uma súbita variação nos níveis de demanda, ocasionada por um aumento ou queda nos níveis de venda do produto. Por exemplo, empresas que negociam commodities estão sempre, em algum momento, sujeitas a instabilidades no fornecimento. Isto se verifica no fato da flutuação dos preços depender do mercado internacional.

Por outro lado, fixando-se $\alpha=0,95$, o objetivo é estabelecer uma política orientada para satisfazer o cliente. Nesse caso, a administração tem como estratégia melhorar ao máximo o nível de serviço quanto ao requisito de entrega do produto, ou seja, realizar entregas no prazo combinado em pelo menos $95 \%$ das vezes. Esta estratégia está ligada a um tipo de produção em que a quantidade em estoque pode ser o grande diferencial. Por exemplo, para empresas que produzem chips de computadores, a pronta entrega é um fator diferencial na consolidação de uma vantagem competitiva.

A Figura 3 mostra as trajetórias de estoque e produção para $\alpha=0,5$ e $\alpha=0,95$. É importante observar que a política de estoque obtida com $\alpha=0,95$ é ligeiramente superior que a obtida com $\alpha=0,5$. Isto significa que para reduzir o risco de falta do produto, a política gerada com $\alpha=0,95$ tende a ser mais conservadora do que a gerada com $\alpha=0,5$. A geração de estoques orientada ao aten-

Tabela 1. Demanda média mensal.

\begin{tabular}{ccccccccccccc}
\hline $\mathbf{k}$ & Jan. & Fev. & Mar. & Abr. & Mai. & Jun. & Jul. & Ago. & Set. & Out. & Nov. & Dez. \\
\hline média & 10 & 11 & 9 & 7 & 6 & 6 & 7 & 8 & 7 & 7 & 8 & 9 \\
\hline
\end{tabular}



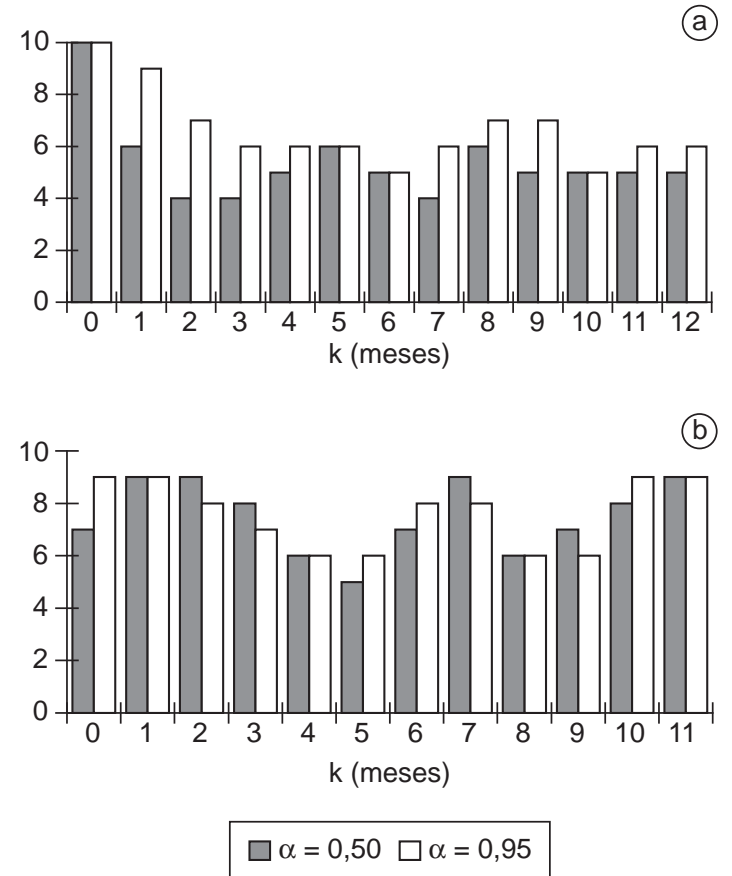

Figura 3. Procedimento OLFC com valores diferentes de $\alpha$. a) estoques $(\mathrm{x})$; e b) produção $(\mathrm{u})$.

dimento futuro da demanda é uma prática muito usada por fornecedores em cadeias de suprimento para minimizar intervenções gerenciais rotineiras devido a fatores não previsíveis de operação como, por exemplo: quebra de máquina, pedidos não esperados, atraso dos fornecedores, flutuações de vendas, etc. A questão é que tal prática eleva os custos de produção e, assim, não constitui surpresa que a política conservadora $\alpha=0,95$ é mais cara que a gerada com $\alpha=0,5$, ou seja, $\mathrm{J}^{*}(\alpha=0,95)=$ $\mathrm{R} \$ 2.120,00$ e $\mathrm{J} *(\alpha=0,5)=\mathrm{R} \$ 1.760,00$. Neste caso, pode-se dizer que o preço pago por aumentar o nível de serviço ao cliente resultou em um aumento no custo total em torno de $20 \%$.

Por fim, é importante observar na Figura 3, que o aumento no nível de serviço (i.e., $\alpha$ próximo de $100 \%$ ) provoca o crescimento dos níveis de estoque ao longo dos períodos. Com isto, a política gerada procura garantir que as expectativas futuras de aumentos de demanda possam ser plenamente atendidas. Assim, empresas que operam em um padrão de produção do tipo flow-shop (HIGGINS et al., 1996), ou seja, empresas formadoras de estoques, podem garantir a confiabilidade de suas entregas. Estas empresas são as principais beneficiadas com o uso de políticas subótimas baseadas no modelo de otimização aqui proposto.

Para analisar a segunda questão, que envolve o conceito de adaptabilidade do procedimento OLFC, considera-se a inclusão neste estudo dos procedimentos malha-aberta (MA) e malha-fechada (MF) (BRYSON; HO, 1975). De modo similar ao OLFC, o procedimento MA é uma abordagem do tipo subótima, Bertesekas (2000). Isto significa que esse procedimento também adota um esquema aproximativo para reduzir a complexidade do problema. A diferença entre essas heurísticas está no uso da informação, o procedimento MA não incorpora nenhuma informação adicional durante a solução do problema equivalente; a única informação permitida refere-se ao nível de estoque inicial, que é conhecido no período $\mathrm{k}=0$ do horizonte de planejamento. Assim, a solução fornecida pela abordagem MA é uma solução estática, ou seja, uma política congelada no tempo. Por outro lado, o procedimento MF gera a solução ótima global para o problema; solução essa que leva em conta todo o espaço de factibilidade gerado pelos conjuntos X e U (SILVA FILHO; CEZARINO, 2004).

Uma vez que, neste exemplo o problema considerado é de pequeno porte, o algoritmo de Programação Dinâmica pode ser utilizado na aplicação dos três procedimentos acima. O principal objetivo é confrontar as três soluções, com respeito aos seus respectivos custos ótimos de operação, visando, assim, confirmar a característica de quase adaptabilidade atribuída ao procedimento OLFC (BERTESEKAS, 2000).

A Figura 4 mostra as trajetórias de estoque e produção geradas pelas abordagens OLFC, MA, e MF, com um nível de satisfação do cliente fixado em $85 \%$ (i.e., $\alpha=0,85$ ). Além disto, no processo de simulação do procedimento OLFC, é considerado que a flutuação da demanda real é igual a demanda média (Tabela 1). Sob esse cenário produtivo, verificou-se que a solução fornecida pela abordagem MA apresenta o pior desempenho. Este comportamento decorre do fato da heurística malha-aberta MA usar, como única informação sobre o sistema, o estado inicial de estoque, ou seja, $\mathrm{x}_{0}=10$. Esta falta de informação sobre o comportamento de sistema de balanço de estoque faz com que a política MA seja essencialmente conservadora quanto ao risco de não atendimento da demanda futura. Com isto, os níveis de estoque para períodos futuros tendem a crescer cumulativamente no tempo, como se observa na Figura 4. Como resultado da aplicação da política MA, obteve-se o custo total de $\mathrm{J}_{\mathrm{MA}}=\mathrm{R} \$ 4.045,00$.

O procedimento ótimo OLFC é também responsável por aumentar os níveis de estoque, porém não de forma cumulativa no tempo como ocorre no procedimento MA. A razão disto é que na abordagem OLFC ocorrem revisões periódicas do plano de produção, num esquema padrão conhecido na literatura como horizonte deslizante (YILDIRIM et al., 2005). O custo ótimo para gerar uma política OLFC para o problema em questão é dado por $\mathrm{J}_{\text {OLFC }}=\mathrm{R} \$ 2.273,00$.

A abordagem MF mantém os níveis de estoque no limite mínimo da capacidade de armazenagem (i.e., $\underline{x}=$ 

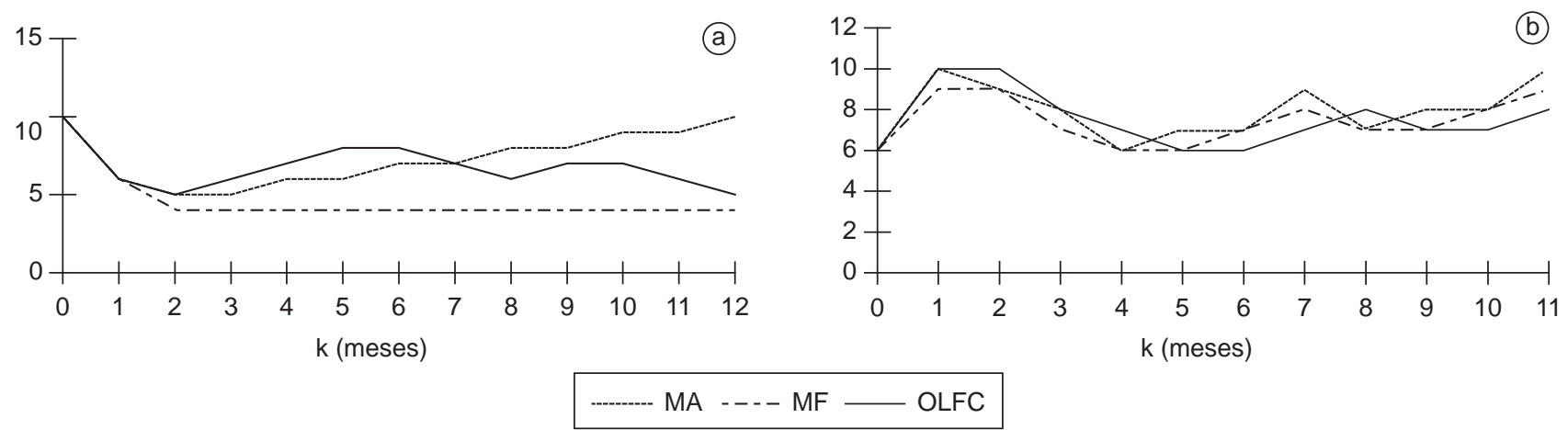

Figura 4. Comparação das políticas OLFC, MA e MF. a) estoque (x); e b) produção (u).

4) ao longo dos períodos do horizonte de planejamento. Esta solução representa a solução ótima global para o problema proposto, considerando sua natureza estocástica. O custo ótimo para aplicar esta política ótima é $\mathrm{J}_{\mathrm{MF}}=\mathrm{R} \$ 1.408,00$.

Por fim, comparando-se os custos obtidos para os três planos, é possível confirmar a propriedade de quase adaptabilidade esperada para a heurística OLFC, isto é, $\mathrm{J}_{\mathrm{MF}}<\mathrm{J}_{\mathrm{OLFC}}<\mathrm{J}_{\mathrm{MA}}$ (BERTESEKAS, 2000).

Com respeito à última questão, será analisado, para efeitos comparativos, o comportamento das três políticas descritas acima. Assim, três casos serão simulados para uma situação mais complexa, cuja a demanda futura é projetada a partir de um modelo de previsão. Nestas circunstâncias, a abordagem MA falha em fornecer uma solução factível para o problema. De fato, observouse durante a simulação a ocorrência de infactibilidade da política MA a partir do período $\mathrm{k}=5$, isto é, $\mathrm{u}(\mathrm{k}) \notin$ $\Omega(\mathrm{x}(\mathrm{k}) ; \alpha=0,85)$ para $\forall \mathrm{k} \geq 5$. Isto novamente se explica pela razão da abordagem MA não usar mecanismos de atualização das informações correntes sobre o sistema de balanço de estoque. Por outro lado, as trajetórias geradas pelos procedimentos OLFC e MF durante o processo de simulação apresentaram-se como soluções factíveis, como ilustrado na Figura 5. O custo ótimo para operar o sistema usando a política malha-fechada MF foi de $\mathrm{J}_{\mathrm{MF}}=\mathrm{R} \$ 1.451,00$; enquanto que o custo OLFC nessa operação foi de $\mathrm{J}_{\mathrm{OLFC}}=\mathrm{R} \$ 2.324,00$.

Finalmente, comparando as trajetórias de estoque e produção para o esquema de simulação acima, é possível concluir que: a) as políticas ótimas de produção obtidas pelas abordagens OLFC e MF estão muito próximas. Isto é um resultado importante, pois a trajetória ilustrada na Figura 5 é uma representação exata de um plano ótimo de produção revisado mensalmente, ou seja, é uma solução ótima dita global do problema proposto neste exemplo; b) as trajetórias de estoques apresentam similares tendências, porém suas magnitudes são completamente diferentes. Os níveis de estoque fornecidos pelo OLFC
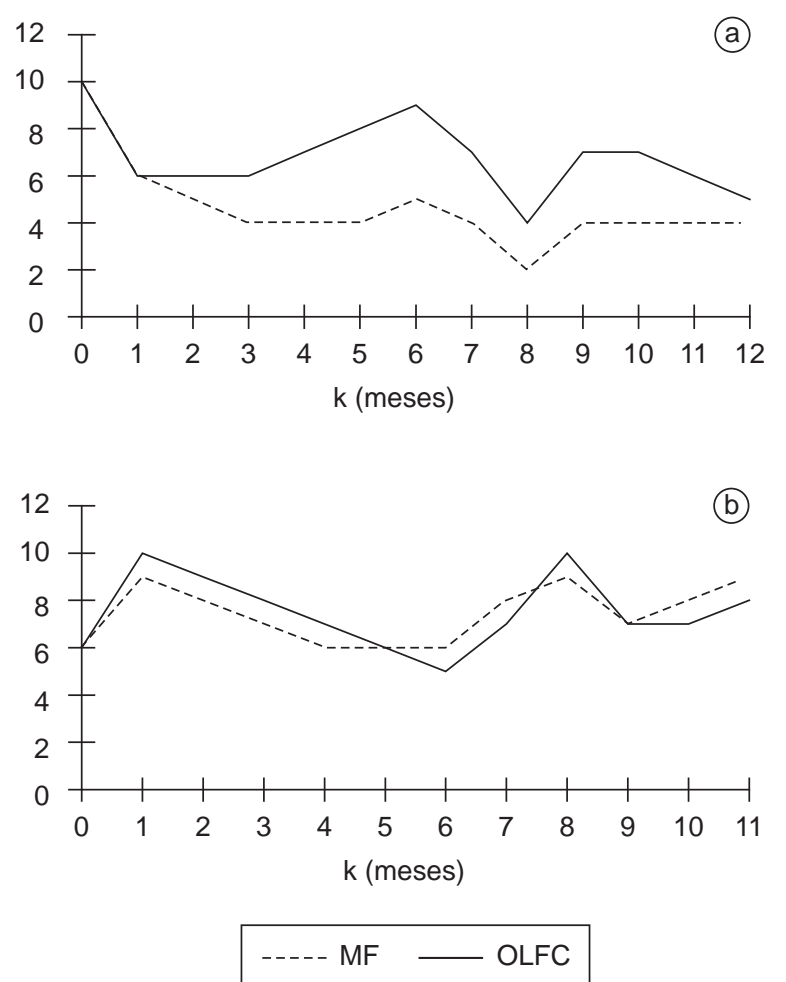

Figura 5. Simulação usando políticas MF e OLFC. a) estoque (x); e b) produção (u).

são maiores que os fornecidos pela abordagem MF. Isto ocorre porque, na aplicação da abordagem MF, todas as possibilidades de obtenção de uma solução ótima global são perseguidas dentro do espaço de restrições do problema. Por outro lado, na aplicação da abordagem OLFC leva-se em conta, para geração da política ótima, apenas a observação do estado corrente de estoque para cada mês k; c) é importante notar que a política MF procura reduzir ao máximo os níveis de estoque e, ao mesmo tempo, manter uma política de produção suavizada (sem flutuações excessivas) de modo a garantir o menor custo de operação. Com isto, durante a simulação pôde-se observar, que para o período $\mathrm{k}=8$, a política $\mathrm{MF}$ usou uma fração do estoque de segurança (i.e., $x(8)=$ 
2) para garantir o atendimento da demanda projetada no mês 4. Esta mesma característica não foi verificada com o uso da política OLFC, a razão disto é que ela utiliza níveis de estoque acima do limite de segurança $\underline{x}=4$, para todos os meses do horizonte de planejamento. $\mathrm{O}$ preço pago, neste caso, é o aumento direto dos custos com armazenagem; e d) a administração pode manipular o índice de desempenho $\alpha$, no sentido de estudar estratégias de atendimento aos clientes, com base na flutuação da demanda projetada. Como resultado, é possível construir diferentes cenários de produção e selecionar aquele que apresente o menor custo sem comprometer as capacidades de operação do sistema.

\section{Conclusão}

Neste trabalho um problema de planejamento agregado da produção, que tem por objetivo a geração de planos de produção, foi formulado a partir de um modelo de controle ótimo estocástico seqüencial, discreto no tempo e com restrições probabilísticas nas variáveis de decisão. Como formulado, o modelo é bastante geral, no sentido de que ele contempla, de forma simultânea, várias características exploradas na literatura.

Devido às dificuldades computacionais para se determinar uma solução ótima global para este problema, um procedimento subótimo conhecido na literatura como open-loop feedback controller (OLFC) foi considerado como abordagem de solução. As vantagens observadas com a aplicação deste procedimento, na geração do plano ótimo de produção, foram as seguintes:

a) ele garante um plano revisado mensalmente que pode gerar uma meta de produção a ser usada como referência nas decisões tomadas pelos administradores nos níveis inferiores da hierarquia de planejamento, como ilustrado na Figura 1;

b) o plano de produção seqüencial pode ser usado pelos administradores para melhorar a qualidade de suas decisões em termos do uso dos recursos indus- triais, haja vista, que o procedimento permite não só revisões mensais como também avaliações de cenário tomando como base o nível de atendimento ao cliente; $\mathrm{e}$

c) tendo em vista as simplificações impostas ao modelo, visando garantir uma implementação computacional viável, é possível aplicar esse procedimento em problemas de grande porte, ou seja, problemas cujo o número de estados de operação do sistema seja superior a três (BERTESEKAS, 2000), fato que inviabiliza o uso do algoritmo de programação dinâmica.

Com o objetivo de analisar as principais características do procedimento OLFC, um exemplo muito simples, mas bastante ilustrativo foi introduzido. A idéia básica foi verificar a influência do índice probabilístico $\alpha$ no nível de serviço apresentado pela solução OLFC, e também comparar a solução OLFC com as apresentadas pelas abordagens malha aberta e malha fechada, de modo a analisar a propriedade de quase adaptabilidade. Assim, através do exemplo, observou-se que a abordagem OLFC apresenta um bom desempenho frente à abordagem malha aberta, embora fique abaixo do desempenho apresentado pela abordagem malha fechada. Com isso, pode-se concluir que o procedimento OLFC é uma abordagem quase adaptativa, sendo assim bastante promissora para lidar com problemas de natureza dinâmica estocástica.

Por fim, é importante destacar futuras pesquisas na área de planejamento e controle da produção, com aplicação deste procedimento a problemas de controle de estoque nos quais não haja informações disponíveis sobre o estado do sistema. Mais recentemente, este tipo de problema vem merecendo interesse de pesquisadores em virtude da necessidade de gerenciamento de estoques à distância em ambientes integrados de cadeias de suprimento. Algumas referências para interessados nesse tema são Aggoun et al. (1999), Bensoussan et al. (2005), Kang e Gershwin (2004), Liberopoulos et al. (2004) e Trethane e Sox (2000). 


\title{
Providing production plans via suboptimal sequential optimization
}

\begin{abstract}
In this paper, the need for developing an aggregate production plan within a hierarchical decision process is discussed. A production planning problem formulated as a sequential stochastic optimal control model with chance constraints is introduced. A global optimal solution is only possible in particular cases with a small number of decision variables. As a consequence, near optimal solutions are usually developed and applied to solve sequential stochastic problems. A suboptimal approach, called Open-Loop Feedback Controller (OLFC), is proposed to solve the aggregate stochastic production planning problem. A simple example is used to evaluate the performance of the OLFC solution computationally in relation to the global (closed-loop) and mean (open-loop) optimal solutions. Quasi-adaptability property of the OLFC procedure is analysed in this example.
\end{abstract}

Keywords: Suboptimal control. Probabilistic models. Dynamic programming. Production costs. Inventory control.

\section{Referências bibliográficas}

AGGOUN, L.; BENKHEROUF, L.; TADJ, L. L. An optimal adaptive estimators for partially observed numbers of defective items in inventory models. Mathematical and Computer Modelling, v. 29, n. 6, p. 83-93, 1999.

ANTHONY, R. N. Planning and control systems: a framework for analysis. Cambridge, MA: Harvard Univ. Press, 1965.

ASTRÖM, K. J. Introduction to stochastic control theory. New York, USA: Academic Press, 1970.

BENSOUSSAN, A.; CAKANYILDIRIM, M.; SETHI, S. P. Partially observed inventory system: The Case of Zero Balance Walk. Technical Report, Management School, University of Texas, 2005. Disponível em: <http://som.utdallas.edu/faculty/ working_papers/SOM200548.pdf>. Acesso em: 28 jul. 2007.

BENSOUSSAN, A.; KLEINDORFER, P. R.; TAPIERO, C. H. S. (eds.). Applied optimal control. Amsterdam, NL: TIMS Studies in the Management Science 9, North-Holland Publishing Company, 1978.

BERTESEKAS, D. P. Dynamic programming and stochastic control. Belmont, MA: Athena Scientific, 2000.

BITRAN, G. R.; YANASSE, H. H. Deterministic approximation to stochastic production problem, Operations Research, v. 32, n. 5, p. 999-1018, 1984

BRYSON, A. E.; HO, Y. Applied optimal control: optimization, estimation and control. Washington D. C: Hemisphere Publishing Corporation, 1975.

CHENG, L.; SUBRAHMANIAN, E, A.; WESTERBERG, W. A comparison of optimal and stochastic programming from a formulation and computation perspective. Computers and Chemical Engineering, v. 29, n. 1, p. 149-164, 2004.

FEIRING, B. R.; SASTRI, T. Improving production planning by utilizing stochastic programming. Computers and Industrial Engineering. v. 19, n. 1-4, p. 53-56, 1990.

GERSHWIN, R. et al. A Control perspective on recent trends in manufacturing systems. Control System Magazine, IEEE, v. 6, n. 2, p. 3-15, 1986.

GRAVES, S. C. A single-item inventory model for a non-stationary demand process. Manufacturing \& Service Operations Management, v. 1, n. 1, 1999.

HACKMAN, S. et al. A stochastic production planning. Technical Report. The Logistic Institute, Georgia Tech and National
University of Singapore, 2002. Disponível em: <www.tliap.nus. edu.sg/Library/download_2.asp?filename=serf-2002-01.pdf $>$. Acesso em: 28 jul. 2007.

HAX, A. C.; CANDEA, D. Production and inventory management. Englewood Cliffs: Prentice-Hall, 1984.

HIGGINS, P.; Le ROY, P.; TIERNEY, L. Manufacturing planning and control: Beyond MRP II, London, UK: Chapman \& Hall, 1996.

HOLT, C. C. et al. Planning Production, Inventory and Work Force. Englewood Cliffs: Prentice-Hall, 1960.

KANG, Y.; GERSHWIN, S. B. Information inaccuracy in inventory systems - stock loss and stockout. Technical Report, Department of Mechanical Engineering, Massachusetts Institute of Technology, 2004. Disponível em: <http://cell1.mit.edu/ papers/kang-gershwin-autoid04.pdf>. Acesso em: 28 jul. 2007.

KLEINDORFER, P. R. et al. Discrete optimal control of production plans. Management Science, v. 22, n. 3, p. 261-273, 1975.

LASSERE, J. B.; BES, C.; ROUBELLAT, F. The stochastic discrete dynamic lot size problem: an open-loop solution. Operation Research, v. 33, n. 2, p. 684-689, 1984.

LEVI, R. et al. Approximation algorithms for stochastic inventory control models. Manufacturing \& Service Operations Management, v. 7, n. 1, p. 81-99, 2005.

LIBEROPOULOS, G. et al. Preface of the stochastic models for the analysis, design, coordination, and control of production inventory systems. Annals of Operations Research, v. 125, n. $1-4$, p. $17-19,2004$

McMillaN, G. K. Tuning and control loop performance. Research Triangle Park, N.C.: Instrument Society of America (An ISA Monograph series), 1983.

MULA, J. et al. Models for production planning under uncertainty: a review. International Journal of Production Economics, v. 103, n. 1, p. 271-285, 2006.

NECK, R. Stochastic control theory and operational research, European Journal of Operational Research, v. 17, n. 3, p. 283-301, 1984.

PAPOULIS, A. Probability, random variables, and stochastic processes. 3. ed. Singapure: McGraw-Hill, 1991.

PEKELMAN, D.; RAUSSER, G. C. Adaptive control: survey of methods and applications. In: BENSOUSSAN, A.; 
KLEINDORFER, P. R.; TAPIERO Ch. S. (eds.) Applied optimal control. Amsterdam, NL: TIMS Studies in the Management Science 9, North-Holland Publishing Company, 1978.

PEREIRA, F. L.; SOUSA, J. B. On the receding horizon hierarchical optimal control of manufacturing systems. Journal of Intelligent Manufacturing, v. 8, n. 5 p. 425-433, 1997

RAO, S.; SCHNELLER IV, G. O. On the stochastic non-sequential production planning problem. Journal of the Operational Research Society, v. 41, n. 3, p. 241-247, 1990.

RANTALA, J.; KOIVISTO, H. Production planning under stochastic time-varying demand: flexible service approach. In: IFAC WORLD CONGRESS, 16., 2006. Praga, República Checa, 2005, Proceedings... (ISBN: 978-0-08-045108-4), Elsevier Science, 2006

SAHINIDIS, N. V. Optimization under uncertainty: state-of-theart and opportunities. Computers \& Chemical Engineering, v. 28 , n. 15 , p. $971-983,2004$

SHEN, R. F. C. Aggregate production planning by stochastic control. European Journal of Operational Research, v. 73, n. 2, pp. 346-359, 1994

SILVA FILHO, O. S.; CEZARINO, W. An optimal production policy applied to a flow-shop manufacturing system. Brazilian Journal of Operations \& Production Management, v. 1, n. 1, 73-92, 2004.
SILVA FILHO, O. S. Estratégias seqüenciais subótimas para planejamento agregado da produção sob incertezas. Gestão \& Produção, v. 7, n. 3, p. 247-268, 2000.

SILVA FILHO, O. S.; VENTURA, S. Optimal feedback control scheme helping managers to adjusting industrial resources of the firm. Control Engineering Practices, v. 7 n. 4, p. 555-563, 1999.

SILVA FILHO, O. S. An aggregate production planning model with demand under uncertainty. Production Planning \& Control, v. 10, n. 8, p. 745-756, 1999.

SILVA FILHO, O. S.; GEROMEL, J. C. Estrutura de controle parcialmente em malha fechada para sistemas lineares estocásticos, Controle \& Automação, v. 2, n. 2, p. 102-108, 1990.

TRETHANE, J. T.; SOX, C. R. Adaptive inventory control for nonstationary demand and partial information, Technical Report. Department of Industrial and Systems Engineering, Auburn University, 2000. Disponível em: <http://www.cba.ua.edu/ $\sim$ csox/NSFAdaptInvy/start.html>. Acesso em: 28 jul. 2007.

VADJA, S. Probabilistic Programming. New York, USA: Academic Press, 1972.

YILDIRIM, I.; TAN, B.; KARAESMEN, F. A multiperiod stochastic production planning and sourcing problem with service level constraints, OR Spectrum, v. 27, n. 2-3, p. 471489, 2005.

\section{Sobre os autores}

\section{Oscar Salviano Silva Filho Wagner Cezarino}

Grupo de Pesquisa em Tecnologias de Gestão Organizacional, Laboratório de Divisão de Gestão Empresarial, Centro de Pesquisas Renato Archer, Rod. D. Pedro I, Km 143,6, CEP 13069-901, Campinas, SP, Brasil, e-mails: oscar.salviano@ cenpra.gov.br; wagner.cezarino@cenpra.gov.br

Agradecimentos: Aos comentários e opiniões abalizadas dos revisores que possibilitaram melhorar a qualidade da apresentação do artigo e ao CNPq pelo apoio através do Processo Integrado de Pesquisa $n^{\circ} .500202 / 2003-6$. 